Pacific Journal of Mathematics

THE ASYMPTOTIC BEHAVIOR OF A FAMILY OF SEQUENCES ANDRew OdLYzKo, PAUL PUDATIE AND BRUCE RezNIC 


\title{
THE ASYMPTOTIC BEHAVIOR OF A FAMILY OF SEQUENCES
}

\author{
P. ERdös, A. Hildebrand, A. OdlyzKo, \\ P. PudAite AND B. RezNick
}

A class of sequences defined by nonlinear recurrences involving the greatest integer function is studied, a typical member of the class being

$$
a(0)=1, \quad a(n)=a(\lfloor n / 2\rfloor)+a(\lfloor n / 3\rfloor)+a(\lfloor n / 6\rfloor) \quad \text { for } n \geq 1 \text {. }
$$

For this sequence, it is shown that $\lim a(n) / n$ as $n \rightarrow \infty$ exists and equals $12 /(\log 432)$. More generally, for any sequence defined by

$$
a(0)=1, \quad a(n)=\sum_{i=1}^{s} r_{i} a\left(\left\lfloor n / m_{l}\right\rfloor\right) \text { for } n \geq 1,
$$

where the $r_{t}>0$ and the $m_{i}$ are integers $\geq 2$, the asymptotic behavior of $a(n)$ is determined.

1. Introduction. Rawsthorne [R] recently asked whether the limit $a(n) / n$ exists for the sequence $a(n)$ defined by

$$
\text { (1.1) } a(0)=1, \quad a(n)=a(\lfloor n / 2\rfloor)+a(\lfloor n / 3\rfloor)+a(\lfloor n / 6\rfloor), \quad n \geq 1,
$$

where $\lfloor x\rfloor$ denotes the greatest integer $\leq x$. If the limit exists, Rawsthorne also asked for its value. We have answered these questions [EHOPR]: the limit exists and equals $12 / \log 432$, where, as in the rest of the paper, log denotes the natural logarithm. Our method leads to a more general result about such recursively defined sequences.

Let $a(n)$ be the sequence defined by

$$
a(0)=1, \quad a(n)=\sum_{i=1}^{s} r_{i} a\left(\left\lfloor n / m_{i}\right\rfloor\right), \quad n \geq 1,
$$

where $r_{i}>0$ and the $m_{i}$ 's are integers $\geq 2$. Let $\tau$ be the (unique) solution to

$$
\sum_{i=1}^{s} \frac{r_{i}}{m_{i}^{\tau}}=1
$$

We distinguish two cases: if there is an integer $d$ and integers $u_{i}$ such that $m_{i}=d^{u_{i}}$, we are in the lattice case, otherwise we are in the ordinary case. In the ordinary case, $\lim a(n) / n^{\tau}$ exists; in the lattice case, $\lim a(n) / n^{\tau}$ does not exist, but $\lim _{k \rightarrow \infty} a\left(d^{k}\right) / d^{k \tau}$ exists. The limit in either case is 
readily computable. The proof involves transforming (1.2) into a renewal equation and using the standard limit theorems for that equation. For a precise statement of our results, see Theorem 2.14 below.

We are interested also in the rapidity of convergence. We prove that $(a(n)-a(n-1)) / n^{\tau}$ is greater than $\gamma \cdot(\log n)^{-(s-1) / 2}$ for some $\gamma>0$ and infinitely many $n$. In Rawsthorne's original sequence (1.1), this result can be strengthened (see Theorem 3.46). For $n=432^{t}$,

$$
\frac{a(n)-a(n-1)}{n} \sim\left(\frac{6}{5 \pi t}\right)^{1 / 2} \text { as } t \rightarrow \infty,
$$

and this is, asymptotically, an upper bound. The numbers $J(m, r):=$ $a\left(2^{m} 3^{r}\right)-a\left(2^{m} 3^{r}-1\right)$ satisfy the so-called "square" functional equation; we use the work of Stanton and Cowan and others to help in the asymptotic analysis.

A somewhat different functional equation was studied by Erdös [E1], [E2]: for $2 \leq a_{1} \leq a_{2} \leq \cdots$ a sequence of integers, let

$$
\begin{aligned}
& F(0)=0, F(1)=1, \\
& F(n)=\sum_{k=1}^{\infty} F\left(\left\lfloor n / a_{k}\right\rfloor\right)+1 \text { for } n>1 .
\end{aligned}
$$

Both the methods and results are different from ours.

2. An application of renewal theory. We fix the following notation. Let integers $m_{i}, 2 \leq m_{i} \leq M$, and positive real numbers $r_{i}$ be given. Define the sequence $a(n)$ recursively by

$$
a(0)=1, \quad a(n)=\sum_{i=1}^{s} r_{i} a\left(\left\lfloor n / m_{i}\right\rfloor\right), \quad n \geq 1 .
$$

For $x \geq 0$ define

$$
A(x)=a(\lfloor x\rfloor)
$$

Since $[x / m n]=[[x / m] / n]$ for positive integers $m$ and $n$, we may define $A(x)$ directly and, in effect, extend the sequence to a function on the positive reals:

(2.3) $A(x)=1$ for $0 \leq x<1, \quad A(x)=\sum_{i=1}^{s} r_{i} A\left(x / m_{i}\right)$ for $x \geq 1$. 
Note that the function $\phi(u)=\sum r_{i} / m_{i}^{u}$ decreases strictly on the real line from $\infty$ to 0 so there exists a unique $\tau>0$ satisfying

$$
\phi(\tau)=\sum_{i=1}^{s} \frac{r_{i}}{m_{i}^{\tau}}:=\sum_{i=1}^{s} p_{i}=1
$$

Now let

$$
f(x)=A(x) / x^{\tau}
$$

so that we may rewrite (2.3) as

$$
\text { (2.6) } f(x)=x^{-\tau}, \quad 0<x<1 ; \quad f(x)=\sum_{i=1}^{s} \frac{r_{i}}{m_{i}^{\tau}} f\left(\frac{x}{m_{i}}\right) \text { for } x \geq 1 \text {. }
$$

Since $p_{i}>0$ and $\sum p_{i}=1, f(x)$ is a convex combination of previous values of $f$ for $x \geq 1$. It is thus unsurprising that $f$ tends to a limit.

It is now appropriate to review some well-known (to probabilists) results about the renewal equation. We paraphrase Feller [F, v. 2, pp. 358-362]. Suppose $h$ is a Riemann integrable function with compact support and $F\{d y\}$ is a probability measure with finite expectation and suppose $g$ satisfies the renewal equation

$$
g(u)=h(u)+\int_{0}^{u} g(u-\nu) F\{d \nu\}, \quad u \geq 0 .
$$

If the mass of $F\{d \nu\}$ is concentrated on a set of the form $\{0, \lambda, 2 \lambda, \ldots\}$, we are in the lattice case; otherwise we are in the ordinary case. The following limit theorem for $g$ is due to Erdös, Feller, and Pollard in the lattice case and Blackwell in the ordinary case.

Renewal Limit Theorem (see [F, v. 2, p. 362]).

(i) In the ordinary case.

$$
\lim _{u \rightarrow \infty} g(u)=\frac{\int_{0}^{\infty} h(u) d u}{\int_{0}^{\infty} y F\{d y\}}
$$

(ii) In the lattice case, let $\lambda$ be chosen to be maximal; then $g$ does not converge, but for any fixed $x \in[0, \lambda)$,

$$
\lim _{n \rightarrow \infty} g(x+n \lambda)=\frac{\lambda \sum_{k=0}^{\infty} h(x+k \lambda)}{\int_{0}^{\infty} y F\{d y\}},
$$

where the limit in (2.9) is taken over integral $n$.

We now return to our problem. Let

$$
g(u)=f\left(e^{u}\right) .
$$


Then (2.6) can be rewritten as

$$
g(u)=\left\{\begin{array}{l}
e^{-\tau u}, \quad u \leq 0 \\
\sum_{i=1}^{s} p_{i} g\left(u-\log m_{i}\right), \quad u \geq 0
\end{array}\right.
$$

Let $F\{d \nu\}$ be the probability measure with mass $p_{i}$ at $\log m_{i}$. Then $g$ satisfies an equation of the form (2.7), where $h$ measures the discrepancy between the full recurrence of (2.11) and that portion provided by the convolution in (2.7). This discrepancy arises from a negative argument of g. Hence,

$$
h(u)=\sum_{u<\log m_{t}} p_{i} e^{-\tau\left(u-\log m_{\imath}\right)}=\sum_{u<\log m_{t}} p_{i} m_{i}^{\tau} e^{-\tau u},
$$

and so

$$
h(u)=\sum_{i=1}^{s} p_{i} m_{i}^{\tau} e^{-\tau u} \chi_{\left[0, \log m_{i}\right)}(u) .
$$

Having now transformed (1.2) into a renewal equation we must decide which case we are in. The mass of $F$ is concentrated at $\left\{\log m_{i}\right\}$, which is a subset of $\{0, \lambda, 2 \lambda, \ldots\}$ for some $\lambda$ (the lattice case) if and only if $m_{i}=d^{u_{i}}$ for some integers $d$ and $u_{i}$. Alternatively, we are in the ordinary case if and only if $\left(\log m_{i}\right) /\left(\log m_{j}\right)$ is irrational for some $\left(m_{i}, m_{j}\right)$.

We now combine these discussions into a theorem.

THEOREM 2.14. Let $a(n)$ be defined by (2.1) and let $\tau$ be defined as above.

(i) If $\tau=0$ then $a(n) \equiv 1$.

(ii) If $\tau \neq 0$ and $\left(\log m_{i}\right) /\left(\log m_{j}\right)$ is irrational for some $\left(m_{i}, m_{j}\right)$ (the ordinary case), then

$$
\lim _{n \rightarrow \infty} \frac{a(n)}{n^{\tau}}=\frac{\sum_{i=1}^{s} p_{i}\left(m_{i}^{\tau}-1\right) / \tau}{\sum_{i=1}^{s} p_{i} \log m_{i}} .
$$

(iii) If $\tau \neq 0$ and $m_{i}=d^{u_{i}}$, where $d$ and the $u_{i}$ 's are integers and $d$ is maximal (the lattice case), then

$$
\lim _{k \rightarrow \infty} \frac{a\left(d^{k}\right)}{d^{k \tau}}=\frac{\sum_{i=1}^{s} p_{i}\left(m_{i}^{\tau}-1\right)}{\sum_{i=1}^{s} p_{i} \log m_{i}} \cdot \frac{d^{\tau} \log d}{d^{\tau}-1} .
$$

Proof. (i) If $\tau=0$, then $\sum r_{i}=1$ and it is easy to see from (2.1) that $a(n) \equiv 1$ by induction. As $u^{\tau}-1 \approx \tau \log u$ for $\tau$ near 0 , this result is consistent with the limiting behavior in (2.15) and (2.16). 
(ii) From our definitions,

$$
\frac{a(n)}{n^{\tau}}=f(n)=g(\log n)
$$

so that information about the limiting behavior of $g(u)$ from the Renewal Limit Theorem can be translated into information about $a(n) / n^{\tau}$. In either the ordinary or lattice case,

$$
\int_{0}^{\infty} y F\{d y\}=\sum_{i=1}^{s} p_{i} \log m_{i}
$$

In the ordinary case, as $\tau \neq 0$, we have by (2.13),

$$
\begin{aligned}
\int_{0}^{\infty} h(u) d u & =\sum_{i=1}^{s} p_{i} m_{i}^{\tau} \int_{0}^{\log m_{i}} e^{-\tau u} d u \\
& =\sum_{i=1}^{s} p_{i} m_{i}^{\tau}\left(1-m_{i}^{-\tau}\right) / \tau .
\end{aligned}
$$

Equation (2.15) follows from the foregoing discussion, (2.8), (2.18), and (2.19).

(iii) The period of the lattice is $\lambda=\log d$, and taking $x=0$ in (2.9),

$$
\begin{aligned}
\sum_{k=0}^{\infty} h(k \lambda) & =\sum_{k=0}^{\infty} h(k \log d) \\
& =\sum_{k=0}^{\infty}\left(\sum_{i=1}^{s} p_{i} m_{i}^{\tau} e^{-\tau k \log d} \chi_{\left[0, \log m_{i}\right)}(k \log d)\right) .
\end{aligned}
$$

Since $m_{i}=d^{u_{i}}$

$$
\begin{aligned}
& \sum_{k=0}^{\infty} e^{-\tau k \log d} \chi_{\left[0, \log m_{l}\right)}(k \log d) \\
& \quad=\sum_{k=0}^{u_{i}-1} e^{-\tau k \log d}=\left(1-d^{-u_{i} \tau}\right) /\left(1-d^{-\tau}\right) \\
&=\left(1-m_{i}^{-\tau}\right) d^{\tau} /\left(d^{\tau}-1\right) .
\end{aligned}
$$

We now exchange the order of summation in (2.20) to obtain

$$
\sum_{k=0}^{\infty} h(k \lambda)=\sum_{i=1}^{s} p_{i} m_{i}^{\tau}\left(1-m_{i}^{-\tau}\right) d^{\tau} /\left(d^{\tau}-1\right)
$$

and (2.16) follows from (2.18), (2.22), and (2.9).

In the lattice case, it is easy to show by induction that the sequence $a(n)$ is constant on intervals of the form $\left[d^{k}, d^{k+1}-1\right]$. For any rational 
$x=j / d^{r}, d^{t}<x<d^{t+1}, a\left(x d^{k}\right)$ is defined for $k \geq r$, and $a\left(x d^{k}\right)=$ $a\left(d^{t-r+k}\right)$. Using (2.16), one can compute $\lim a\left(d^{k} x\right) /\left(d^{k} x\right)^{\tau}$; we omit the details.

As a check of Theorem 2.14, consider the following simple lattice example with $s=1$ :

$$
a(0)=1 ; \quad a(n)=d^{\alpha} a(\lfloor n / d\rfloor), \quad n \geq 1 .
$$

It is easy to see in this case that $\tau=\alpha$ and $a\left(d^{k}\right)=d^{(k+1) \alpha}$, so that $a\left(d^{k}\right) / d^{k \tau} \equiv d^{\alpha}$. Substituting $p_{1}=1, m_{1}=d$, and $\tau=\alpha$ into (2.16) returns $d^{\alpha}$, as predicted.

It is perhaps worth mentioning that the existence of $\lim _{n \rightarrow \infty} f(n)$ can be proved without recourse to the Renewal Limit Theorem. Here is a sketch of the argument, without proofs. First, from (2.6), $\alpha \geq f(x) \geq \beta$ for $x \in[y, M y]$, where $y \geq 1$ and $M \geq \max m_{i}$ implies that $\alpha \geq f(x) \geq \beta$ for all $x \geq y$. Thus $L=\varlimsup \lim f(x)$ and $l=\lim f(x)$ are positive and finite. Pick sequences $r_{k} \rightarrow \infty$ and $s_{k} \rightarrow \infty$ with $f\left(r_{k}\right) \rightarrow L, f\left(s_{k}\right) \rightarrow l$ and $r_{k}<s_{k}<M r_{k}$. The next step in the argument is proved as in $\S 3 ; a(n) \neq$ $a(n-1)$ if and only if $n=m_{1}^{e_{1}} \cdots m_{s}^{e_{s}}$ for some integers $e_{i}$ and $\tau \neq 0$, $a(n) \geq a(n-1)$ if $\tau>0$ and $a(n) \leq a(n-1)$ if $\tau<0$. There is a dichotomy depending on which case arises. In the lattice case, $a(n)$ is constant on intervals $\left[d^{k}, d^{k+1}-1\right]$ and the substitutions $m=d^{u_{2}}, b(k)$ $=a\left(d^{k}\right)$ show that $\{b(k)\}$ satisfies a linear difference equation for $k$ sufficiently large. By the standard method for solving linear difference equations (see [T, Ch. 4], for example) $a\left(d^{k}\right)=b(k)=c \cdot \beta^{k}+o\left(\beta^{k}\right)=$ $c d^{k \tau}+o\left(d^{k \tau}\right)$ for appropriate constants. (See the discussion following Corollary 3.12 for more details.)

In the ordinary case, suppose $\left(\log m_{i}\right) /\left(\log m_{j}\right) \notin Q$ and let $m=m_{i}$ and $\bar{m}=m_{j}$. For any $\varepsilon>0$ there exists $E$ so that every $x$ in $\left[M^{-1}, M\right]$ is contained in an interval $[w, w(1+\varepsilon)]$, where $w=m^{e_{1}} / \bar{m}^{e_{2}}$ and $e_{1}$ and $e_{2}$ are positive integers $\leq E$. Let $W$ be any finite set of integers of the form $m_{1}^{f_{1}} \cdots m_{s}^{f_{s}}$; for $k$ sufficiently large and any $w \in W, f\left(r_{k} / w\right)$ is close to $L$ and $f\left(s_{k} / w\right)$ is close to $l$. (This is proved by induction; basically, if a weighted average like (2.6) is close to the maximum then its components can't be too far off.) Now suppose $\tau>0$ and $L>l$ and let $x=s_{k} / r_{k}$, where $k$ is sufficiently large; let $w=m^{e_{1}} / \bar{m}^{e_{2}}$ be chosen so that $x \in$ $[w, w(1+\varepsilon)]$. Then $r^{\prime}=r_{k} / \bar{m}^{e_{2}}$ is a little less than $s^{\prime}=s_{k} / m^{e_{1}}$, but $f\left(r^{\prime}\right)$ is close to $L$ and $f\left(s^{\prime}\right)$ is close to $l$. As $\tau>0, a\left(s^{\prime}\right) \geq a\left(r^{\prime}\right)$, and this gives a contradiction to $L>l$. (More precisely, $\varepsilon$ is chosen so that $L-\varepsilon>$ $(1+\varepsilon)^{\tau}(l+\varepsilon)$.) A similar contradiction can be wrought when $\rho<0$. In either case, $L=l$ so the limit exists. This method, although self-contained, gives no hint about the actual value of the limit. 
3. Rates of convergence. We retain the notation of the last section and continue to assume that $a(n)$ is defined by (2.1). Let

$$
J(n)=a(n)-a(n-1)
$$

denote the jump of the sequence at $n$. In this section we derive closed forms for $a(n)$ and $J(n)$ and use them to give an indication of the rate of convergence of $f$. Ideally, one would discuss the behavior of $|f(x)-\lim f(x)|$. As a step in that direction, we consider the "jumps" of $f$. It is clear from (2.2) and (2.5) that $f$ is everywhere continuous from the right and $f$ is continuous from the left except possibly at certain integers. Let

(3.2) $z(n)=f(n)-\lim _{\varepsilon \rightarrow 0^{+}} f(n-\varepsilon)=\frac{a(n)-a(n-1)}{n^{\tau}}=\frac{J(n)}{n^{\tau}}$.

We shall show in this section that, in the ordinary case, $|z(n)|>$ $c(\log n)^{-(s-1) / 2}$ for some $c>0$ and infinitely many integers $n$. In Rawsthorne's original problem, (1.1), the exponent of $\log n$ may be improved from -1 to $-1 / 2$.

In finding a closed form for $a(n)$, the following notation is useful. Let $\mathbf{i}=\left(i_{1}, \ldots, i_{l}\right), l \geq 1$, be an $l$-tuple of integers, $1 \leq i_{J} \leq s$. Let $I(\mathbf{i})$ be the associated interval:

$$
I(\mathbf{i})=\left[m_{i} \cdots m_{t_{l-1}}, \ldots, m_{l} \cdots m_{i_{l-1}} m_{t_{l}}\right) .
$$

(If $l=1$ in (3.3), take the left-hand endpoint to be 1.) As an inverse function to $I$, for $x \geq 1$, let

$$
B(x)=\{\mathbf{i}: x \in I(\mathbf{i})\} .
$$

THEOREM 3.5. For $x \geq 1$.

$$
A(x)=\sum_{\mathbf{i} \in B(x)} r_{i_{1}} \cdots r_{i_{i}} .
$$

Proof. Recall the basic recurrence (2.3):

$$
A(x)=\sum_{i=1}^{s} r_{i} A\left(x / m_{l}\right) \text {. }
$$

Consider the infinite tree with root " $x$ " and valence $s$ so that each node " $y$ " on the $k$ th level is connected to the nodes " $y / m_{l}$ ", $1 \leq i \leq s$ on the $(k+1)$ st level. We use this tree to iterate the recurrence (2.3) until the argument of $A$ goes below 1 for the first time. In this way, the path from $x$ to $x / m_{i_{1}}, \ldots$, to $x /\left(m_{i_{1}} \cdots m_{i_{l}}\right)$ acquires the coefficient $r_{i_{1}} \cdots r_{i_{l}}$. Since $\mathbf{i}=\left(i_{1}, \ldots, i_{l}\right)$ is in $B(x)$ by construction and $A\left(x / \Pi m_{j}\right)=1,(3.6)$ is established.

We now derive a recurrence for $J(n)=a(n)-a(n-1)$ and find a closed form for $J(n)$. 
THEOREM 3.7.

(i)

(ii)

$$
J(n)=\sum_{m_{\imath} \mid n} r_{i} J\left(n / m_{l}\right)
$$

$$
J(n)=\left(\sum_{i=1}^{s} r_{i}-1\right) \sum_{m_{1}^{e_{1}} \cdots m_{s}^{e_{s}}=n} \frac{\left(e_{1}+\cdots+e_{s}\right) !}{e_{1} ! \cdots e_{s} !} r_{1}^{e_{1}} \cdots r_{s}^{e_{s}}
$$

Proof. (i) We have from (2.3)

$$
J(n)=\sum_{i=1}^{s} r_{i}\left(A\left(\frac{n}{m_{l}}\right)-A\left(\frac{n-1}{m_{l}}\right)\right) .
$$

If $m_{l}+n$ then $\left\lfloor n / m_{i}\right\rfloor=\left\lfloor(n-1) / m_{i}\right\rfloor$ so the $i$ th term is zero; if $m_{l} \mid n$, then by definition, the $i$ th term is $r_{i} J\left(n / m_{i}\right)$.

(ii) Observe that $J(1)=a(1)-a(0)=\sum_{i=1}^{s} r_{i}-1$. Then, consider each representation of $n$ as a product $m_{1}^{e_{1}} \cdots m_{s}^{e_{s}}$. The formula (ii) follows by induction from (i) and the well-known multinomial recurrence:

$$
\frac{\left(e_{1}+\cdots+e_{s}\right) !}{e_{1} ! \cdots e_{s} !}=\sum_{e_{1} \geq 1} \frac{\left(e_{1}+\cdots+e_{s}-1\right) !}{e_{1} ! \cdots\left(e_{i}-1\right) ! \cdots e_{s} !} .
$$

We note that (ii) can also be derived from Theorem 3.5 and a consideration of $B(n)-B(n-1)$ and $B(n-1)-B(n)$. If we consider the representations $n=m_{1}^{e_{1}} \cdots m_{s}^{e_{s}}$ as formally distinct, we may let $j\left(f_{1}, \ldots, f_{s}\right)$ denote that portion of the jump $J(n)$ contributed by the representation $n=m_{1}^{f_{1}} \cdots m_{s}^{f_{s}}$. In view of Theorem 3.7 we have the following recurrences and generating function.

$$
\begin{aligned}
& \left\{\begin{array}{l}
j\left(e_{1}, \ldots, e_{s}\right)=\sum_{e_{i} \geq 1} r_{l} j\left(e_{1}, \ldots, e_{i}-1, \ldots, e_{s}\right), \\
j(0, \ldots, 0)=\sum_{i=1}^{s} r_{i}-1 .
\end{array}\right. \\
& j\left(e_{1}, \ldots, e_{s}\right)=\left(\sum_{i=1}^{s} r_{i}-1\right) \frac{\left(e_{1}+\cdots+e_{s}\right) !}{e_{1} ! \cdots e_{s} !} r_{1}^{e_{1}} \cdots r_{s}^{e_{s}} . \\
& \mathscr{J}\left(z_{1}, \ldots, z_{s}\right)=\sum j\left(e_{1}, \ldots, e_{s}\right) z_{1}^{e_{1}} \cdots z_{s}^{e_{s}} \\
& =\left(\sum_{i=1}^{s} r_{i}-1\right)\left(1-\sum_{i=1}^{s} r_{i} z_{i}\right)^{-1} \text {. }
\end{aligned}
$$

Corollary 3.12. If $\tau>0$ then $J(n)>0$ at all $n$ of the form $m_{1}^{e_{1}} \cdots m_{s}^{e_{s}} ;$ if $\tau<0$ then $J(n)<0$ at all such $n$. 
Proof. From Theorem 3.7 (ii), the sign of $J(n)$ is the sign of $\sum_{i=1}^{s} r_{i}-1$, which equals $\phi(0)-\phi(\tau)$ in the notation of (2.4). Since $\phi$ is strictly decreasing, the conclusions follow immediately.

We now turn our attention to the size of $z(n)$. It is convenient to dispose of the lattice case. As $f\left(d^{k}\right)$ converges to a limit $l$ and $A(x)$ is constant on $\left[d^{k-1}, d^{k}\right), z\left(d^{k}\right) \sim l\left(1-d^{-\tau}\right)$. It is more interesting to look at $f\left(d^{k+1}\right)-f\left(d^{k}\right)$. Let $m_{i}=d^{u_{i}}$ and let $u=\max u_{i}$. Then from (2.6),

$$
f\left(d^{k}\right)=\sum_{i=1}^{s} p_{l} f\left(d^{k-u_{i}}\right) .
$$

Let $\psi(t)=t^{u}-\sum p_{i} t^{u-u_{t}}$ be the characteristic equation of the linear recurrence satisfied by $f\left(d^{k}\right)$. Clearly $\psi(1)=0$ and, as $\lim \left|f\left(d^{k}\right)\right|<\infty$, it follows that the other roots of $\psi$ have moduli less than one. Hence there exists a polynomial $q$ of degree at most $s-1$ and $\lambda, 0 \leq \lambda<1$ so that

$$
\left|f\left(d^{k}\right)-1\right| \leq q(k) \lambda^{k}+o\left(\lambda^{k}\right) .
$$

It follows that $\left|f\left(d^{k+1}\right)-f\left(d^{k}\right)\right| \leq c k^{r} \lambda^{k}$ for sufficiently large $k, r \leq$ $s-1$ and some $c>0$.

Henceforth we assume the ordinary case and $\tau \neq 0$. We first need two approximation lemmas. The first follows directly from the Stirling approximation $\Gamma(w+1) \sim w^{w} e^{-w} \sqrt{2 \pi w}$ and we omit the proof. The second allows us to adjust from real numbers to integers in our asymptotic analysis.

LEMMA 3.15. Fix $\alpha_{i}>0, \sum_{i=1}^{s} \alpha_{i}=1$ and define

$$
F\left(x_{1}, \ldots, x_{s}\right)=\frac{\Gamma\left(\sum_{i=1}^{s} x_{i}+1\right)}{\prod_{i=1}^{s} \Gamma\left(x_{i}+1\right)} .
$$

Then, as $q \rightarrow \infty$

$$
F\left(\alpha_{1} q, \ldots, \alpha_{s} q\right) \sim(2 \pi q)^{-(s-1) / 2} \prod_{i=1}^{s} \alpha_{i}^{-\left(\alpha_{i} q+1 / 2\right)} .
$$

LEMMA 3.18. Fix $\alpha_{i}>0, \sum_{i=1}^{s} \alpha_{i}=1$ and define

$$
\phi\left(q ; t_{1}, \ldots, t_{s}\right)=\frac{F\left(\alpha_{1} q+t_{1}, \ldots, \alpha_{s} q+t_{s}\right)}{F\left(\alpha_{1} q, \ldots, \alpha_{s} q\right)} .
$$

Then there exists $c>0$ so that for all sufficiently large $q$ and all choices of $t_{i}$ with $\left|t_{i}\right|<1$ and $\sum_{i=1}^{s} t_{i}=0$,

$$
c^{-1}<\phi\left(q ; t_{1}, \ldots, t_{s}\right)<c .
$$


Proof. From (3.16) we have

$$
\text { (3.21) } \begin{aligned}
\phi\left(q ; t_{1}, \ldots, t_{s}\right) & =\frac{\Gamma(q+1)}{\prod_{i=1}^{s} \Gamma\left(\alpha_{i} q+t_{i}+1\right)} / \frac{\Gamma(q+1)}{\prod_{i=1}^{s} \Gamma\left(\alpha_{i} q+1\right)} \\
& =\prod_{i=1}^{s} \frac{\Gamma\left(\alpha_{i} q+1\right)}{\Gamma\left(\alpha_{i} q+t_{i}+1\right)} .
\end{aligned}
$$

Let

$$
H(\alpha, q, t)=\log \Gamma(\alpha q+t+1)-\log \Gamma(\alpha q+1) .
$$

As $\log \Gamma$ is convex, for $|t|<1, t \neq 0$ we have

$$
\begin{aligned}
& \log \Gamma(\alpha q+2)-\log \Gamma(\alpha q+1) \\
& \quad \geq \frac{H(\alpha, q, t)}{t} \geq \log \Gamma(\alpha q+1)-\log \Gamma(\alpha q),
\end{aligned}
$$

hence $H(\alpha, q, t)=t \log (\alpha q+p), 0 \leq p \leq 1$, and

$$
\begin{array}{r}
\text { (3.24) }-\log \phi\left(q ; t_{1}, \ldots, t_{s}\right)=\sum_{i=1}^{s} H\left(\alpha_{i}, q, t_{l}\right)=\sum_{i=1}^{s} t_{i} \log \left(\alpha_{i} q+p_{i}\right) \\
=\sum_{i=1}^{s} t_{l} \log \alpha_{i}+\sum_{i=1}^{s} t_{i} \log q+\sum_{i=1}^{s} t_{i} \log \left(1+\frac{p_{l}}{\alpha_{i} q}\right)
\end{array}
$$

Since $\sum t_{l}=0,\left|t_{i}\right|<1$ and $\left|p_{i}\right|<1$,

$$
\left|\log \phi\left(q ; t_{1}, \ldots, t_{s}\right)\right| \leq \sum_{i=1}^{s} t_{i} \log \alpha_{i}+\sum_{i=1}^{s} \frac{1}{\alpha_{i} q},
$$

from which (3.20) follows.

THEOREM 3.26. In the ordinary case with $\tau \neq 0$ there exists $\gamma>0$ so that

$$
|z(n)|>\gamma \cdot(\log n)^{-(s-1) / 2}
$$

for infinitely many $n$.

Proof. The main idea is to let $n=\left(m_{1}^{p_{1}} \cdots m_{s}^{p_{s}}\right)^{q}$ for large $q$. Large in this case means that (3.17) is a good approximation. Since $p_{i} q$ is not an integer in general, we need the approximation of Lemma 3.18.

To be specific, choose $q$ large and choose integers $e_{i}, \sum_{i=1}^{s} e_{i}=q$ such that $\left|e_{i}-p_{i} q\right|<1$ for all $i$. By Theorem 3.7 (ii), we may ignore other representations of $n$ and

$$
|J(n)| \geq \alpha \cdot \frac{\left(e_{1}+\cdots e_{s}\right) !}{e_{1} ! \cdots e_{s} !} r_{1}^{e_{1}} \cdots r_{s}^{e_{s}}
$$


where $\alpha=\left|\sum_{i=1}^{s} r_{i}-1\right|=|\phi(\tau)-\phi(0)|>0$. We now replace $e_{i}$ by $p_{i} q$ in (3.28): $\Pi r_{i}^{e_{i}}$ changes by a bounded factor, and by Lemma 3.18 we have

$$
|J(n)| \geq \beta \cdot \frac{\Gamma(q+1)}{\prod_{i=1}^{s}\left(p_{i} q+1\right)} \cdot \prod_{i=1}^{s} r_{i}^{p_{i} q},
$$

where $\beta$ has absorbed all other constants. Finally, by Lemma 3.16,

$$
\begin{aligned}
|J(n)| & \geq \beta \cdot(2 \pi q)^{-(s-1) / 2} \prod_{i=1}^{s} p_{i}^{-\left(p_{i} q+1 / 2\right)} r_{i}^{p_{i} q}(1-\varepsilon) \\
& =\gamma q^{-(s-1) / 2} \prod_{i=1}^{s}\left(r_{i} / p_{i}\right)^{p_{i} q} \\
& =\gamma q^{-(s-1) / 2} \prod_{i=1}^{s} m_{i}^{\tau p_{i} q}=\gamma q^{-(s-1) / 2} n^{\tau} .
\end{aligned}
$$

Since $z(n)=J(n) / n^{\tau}$ and $q=(\log n) /\left(\sum p_{i} \log m_{i}\right),(3.27)$ follows.

It is possible to sharpen the constant slightly by noting that for any $\varepsilon>0$ there are infinitely many $q$ such that $p_{i} q$ is within $\varepsilon$ of an integer for all $i$. (Standard pigeonhole principle argument.) If $s$ were to equal 1 then (3.27) would violate the convergence of $f$, except that $s=1$ is always a lattice case.

We conclude this paper by returning to Rawsthorne's original problem:

$$
a(0)=1, \quad a(n)=a(\lfloor n / 2\rfloor)+a(\lfloor n / 3\rfloor)+a(\lfloor n / 6\rfloor) .
$$

By Theorem 3.7 we know that $a(n)$ jumps only at numbers of the form $2^{e_{1}} 3^{e_{2}} 6^{e_{3}}$; that is, products of 2 and 3 . Let

$$
J(m, r)=: J\left(2^{m} 3^{r}\right) .
$$

Then $m=e_{1}+e_{3}, r=e_{2}+e_{3}$, and by both parts of Theorem 3.7,

$$
\begin{aligned}
& J(m, r)=2 \sum_{i} \frac{(m+r-i) !}{(m-i) !(r-i) ! i !}=2 \sum_{i}\left(\begin{array}{c}
m+r-i \\
m-i
\end{array}\right)\left(\begin{array}{c}
r \\
i
\end{array}\right), \\
& \left\{\begin{array}{lr}
J(m, r)=J(m, r-1)+J(m-1, r)+J(m-1, r-1), \\
J(0,0)=J(m, 0)=J(0, r)=2 .
\end{array}\right.
\end{aligned}
$$

Unsurprisingly, such a simply defined recurrence has a large literature; (3.33) is called the "square" functional equation and arises as a natural generalization of Pascal's triangle. (Actually, $\frac{1}{2} J$ is the standard form.) The first problem on the 19th Putnam Competition to show that 
$S(n)=\frac{1}{2} \sum_{i} J(i, n-i)$ satisfies the recurrence $S(n+2)=2 S(n+1)+$ $S(n)$ [GGK, p. 53]). This recurrence then arose in Golomb's study of sphere packing in the Lee metric [Go]. Stanton and Cowan [SC] considered (3.32) in its own right and were the first to prove Lemma 3.34 below. A. K. Gupta [Gu1] [Gu2] gave different proofs and generalized these numbers further, as did Carlitz [Ca] and Alladi and Hoggatt [AH]. The function $\frac{1}{2} J$ has a natural interpretation as the number of ways to go from $(0,0)$ to $(m, r)$ with steps of size $(1,0),(0,1)$ or $(1,1)$; see Fray and Roselle [FR] or Handa and Mohanty [HM]. Greene and Knuth [GK; pp. 111-113] discuss the asymptotics of $J(m, m)$.

Our analysis of $z\left(2^{m} 3^{r}\right)$ relies crucially on the following combinatorial lemma.

LEMMA 3.34.

$$
J(m, r)=2 \sum_{i}\left(\begin{array}{c}
m \\
i
\end{array}\right)\left(\begin{array}{c}
r \\
i
\end{array}\right) 2^{i}=2 \sum_{i}\left(\begin{array}{c}
m+r-i \\
m-i
\end{array}\right)\left(\begin{array}{c}
r \\
i
\end{array}\right) .
$$

Proof. Consider the coefficient of $x^{m}$ in

$$
2(1+x)^{m}(1+2 x)^{r}=2 \sum_{i}\left(\begin{array}{l}
r \\
i
\end{array}\right) x^{i}(1+x)^{m+r-i} .
$$

Stanton and Cowan originally proved this lemma by a sequence of standard combinatorial substitutions. Gupta used a number of methods, including the following hypergeometric representation [Gu, Lemma 4]:

$$
\frac{1}{2} J(m, r)={ }_{2} F_{1}(-m,-r ; 1,2) .
$$

Lemma 3.34 leads to a natural probabilistic interpretation of $J(m, r)$. Let

$$
\alpha(m, i)=\frac{\left(\begin{array}{c}
m \\
i
\end{array}\right)}{2^{m}}, \quad \beta(r, i)=\frac{\left(\begin{array}{c}
r \\
i
\end{array}\right) 2^{i}}{3^{r}} .
$$

These denote the probabilities of $i$ successes in $m$ and $r$ Bernoulli trials with $p=\frac{1}{2}$ and $\frac{2}{3}$ respectively. As

$$
z\left(2^{m} 3^{r}\right)=\frac{J(m, r)}{2^{m} 3^{r}}=2 \sum_{i} \alpha(m, i) \beta(r, i),
$$

one expects $z\left(2^{m} 3^{r}\right)$ to be largest when the probability distributions peak simultaneously; that is, when $m / 2 \approx 2 r / 3$, cf. Proposition 3.41. As a preliminary bound, note that $\alpha(m, i) \leq \alpha(m, m / 2)$ and $\beta(r, i) \leq$ $\beta(r, 2 r / 3)$, replacing factorials by $\Gamma$ as necessary. By Lemma 3.15, 
$\alpha(m, i) \leq \gamma_{0} m^{-1 / 2}$ and $\beta(r, i) \leq \gamma_{1} r^{-1 / 2}$ for appropriate $\gamma_{i}>0$. Hence

$$
z\left(2^{m} 3^{r}\right) \leq \min \left(\gamma_{0} m^{-1 / 2}, \gamma_{1} r^{-1 / 2}\right) \text {. }
$$

Since $\log \left(2^{m} 3^{r}\right)=m \log 2+r \log 3,(3.39)$ implies that $z(n) \leq \gamma(\log n)^{-1 / 2}$ for some $\gamma>0$ and all $n$.

Consider now the normal approximation to the binomial distribution, see e.g. [F, v. 1, p. 170]. For fixed $k$,

$$
\left\{\begin{array}{l}
\alpha\left(m, \frac{m}{2}+k \frac{\sqrt{m}}{2}\right) \sim \frac{2}{\sqrt{m}} \frac{1}{\sqrt{2 \pi}} e^{-k^{2} / 2}, \\
\beta\left(r, \frac{2 r}{3}+k \frac{\sqrt{2 r}}{3}\right) \sim \frac{3}{\sqrt{2 r}} \frac{1}{\sqrt{2 \pi}} e^{-k^{2} / 2} .
\end{array}\right.
$$

Let $\Delta(m, r)=|m / 2-2 r / 3|$. We now show that if $\Delta(m, r)$ is comparable to $\sqrt{m}$ and $\sqrt{r}$, then $z\left(2^{m} 3^{r}\right)$ is quite a bit smaller than $\gamma(\log n)^{-1 / 2}$.

Proposition 3.41. Fix $k, \varepsilon>0$ and suppose

$$
\Delta(m, r)=\left|\frac{m}{2}-\frac{2 r}{3}\right|>k\left(\frac{\sqrt{m}}{2}+\frac{\sqrt{2 r}}{3}\right) .
$$

Then for sufficiently large $m$ and $r$,

$$
z\left(2^{m} 3^{r}\right) \leq 2(1+\varepsilon)\left(\frac{2}{\sqrt{m}}+\frac{3}{\sqrt{2 r}}\right) \frac{1}{\sqrt{2 \pi}} e^{-k^{2} / 2} .
$$

Proof. If (3.42) holds, then for each $i$ at least one of the inequalities

$$
\left|\frac{m}{2}-i\right|>\frac{k \sqrt{m}}{2} \text { or }\left|\frac{2 r}{3}-i\right|>\frac{k \sqrt{2 r}}{3}
$$

is valid. Suppose that $m$ and $r$ are large enough that the approximation in (3.40) becomes an inequality after multiplication by $1+\varepsilon$. Let

$$
I=\left\{i:\left|\frac{m}{2}-i\right|>\frac{k \sqrt{m}}{2}\right\}
$$

then

$$
\begin{aligned}
z\left(2^{m} 3^{r}\right) \leq & 2(1+\varepsilon)\left(\sum_{i \in I} \alpha(m, i)\right) \frac{2}{\sqrt{m}} \frac{1}{\sqrt{2 \pi}} e^{-k^{2} / 2} \\
& +2(1+\varepsilon)\left(\sum_{i \notin I} \beta(r, i)\right) \frac{3}{\sqrt{2 r}} \frac{1}{\sqrt{2 \pi}} e^{-k^{2} / 2} \\
\leq & 2(1+\varepsilon)\left(\frac{2}{\sqrt{m}}+\frac{3}{\sqrt{2 r}}\right) \frac{1}{\sqrt{2 \pi}} e^{-k^{2} / 2}
\end{aligned}
$$


We remark that, if $r \sim \alpha m$, where $\alpha \neq 3 / 4$, then this proposition implies that

$$
z(n)=z\left(2^{m} 3^{r}\right) \leq h_{1}(\alpha)(\log n)^{-1 / 2} n^{-h_{2}(\alpha)},
$$

where $h_{1}(\alpha)$ and $h_{2}(\alpha)$ are complicated positive algebraic functions of $\alpha$. We spare the reader the gory details. The asymptotic behavior of

$$
\sum\left(\begin{array}{c}
c \\
i
\end{array}\right)\left(\begin{array}{c}
\alpha c \\
i
\end{array}\right) x^{i}
$$

has been studied by Laquer [La]; more precise information than Proposition 3.41 can be found there, as can our final estimate, whose proof we sketch.

THEOREM 3.46. For $n=432^{t}=2^{4 t} 3^{3 t}$,

$$
z(n) \sim\left(\frac{6}{5 \pi t}\right)^{1 / 2}=\left(\frac{6 \log 432}{5 \pi \log n}\right)^{1 / 2} .
$$

Proof. After a reindexing, (3.38) becomes

$$
z(n)=\sum_{i} \alpha(4 t, 2 t+i) \beta(3 t, 2 t+i) .
$$

By Feller [v. 1, p. 170], the estimates (3.40) are valid for $|i| \leq t^{2 / 3-\varepsilon}$, so the tails can be ignored. These approximations reduce to a Riemann sum:

$$
z(n) \sim \sqrt{\frac{3}{2}} \frac{1}{\pi} \frac{1}{\sqrt{t}} \sum_{i} e^{-5 i^{2} / 4 t} \sim \sqrt{\frac{6}{5 \pi t}} .
$$

As a measure of the slowness of convergence of $f$ and the accuracy of (3.47), let $n=432^{5} \simeq 1.5 \times 10^{13}$. Then $f(n-1) \simeq 1.8430, f(n) \simeq$ 2.1175 , so $z(n) \simeq .2745$, whereas $(6 /(25 \pi))^{1 / 2} \simeq .2764$.

\section{REFERENCES}

[AH] K. Alladi and V. Hoggatt, Jr., On Tribonacci numbers and related functions, Fibonnaci Quart., 15 (1977), 42-45.

[Ca] L. Carlitz, Some q-analogues of certain combinatorial numbers, SIAM J. Math. Anal., 4 (1973), 433-436.

[E1] P. Erdös, On some asymptotic formulas in the theory of 'Factorisatio numerorum', Annals Math., 42 (1941), 989-993.

[E2] Corrections to two of my Papers, (with an appendix by E. Hille), Annals Math., 44 (1943), 647-651.

[EHOPR] P. Erdös, A. Hildebrand, A. Odlyzko, P. Pudaite, and B. Reznick, A very slowly converging sequence, Math. Mag., 58 (1985), 51-52. 
W. Feller, An Introduction to Probability Theory and its Applications, 2nd ed., Wiley, New York, vol. I, 1957, vol. II, 1971.

[FR] R. D. Fray and D. Roselle, Weighted lattice paths, Pacific J. Math., 37 (1971), 85-96.

[GGK] A. M. Gleason, R. E. Greenwood, and L. M. Kelly, The William Lowell Putnam Mathematical Competition, Math. Assn. of America, 1980.

[Go] S. Golomb, Sphere Packing, Coding Metrics and Chess Puzzles, in Proc. 2nd Chapel Hill Conference on Combinatorial Mathematics, (1970), 176-189.

[GK] D. H. Greene and D. E. Knuth, Mathematics for the Analysis of Algorithms, 2nd ed., Birkhäuser, Boston, 1982.

[Gu1] A. K. Gupta, On a 'square' functional equation, Pacific J. Math., 50 (1974), 449-454.

[Gu2] _ _ Generalisation of a 'Square' Functional Equation, Pacific J. Math., 57 (1975), 419-422.

[HM] B. R. Handa and S. G. Mohanty, Higher dimensional lattice paths with diagonal steps, Discrete Math., 15 (1976), 137-140.

[La] H. D. Laquer, Asymptotic limits for a two-dimensional recursion, Stud. Appl. Math., 64 (1981), 271-277.

[R] D. Rawsthorne, Problem 1185, Math. Mag. 57 (1984), 42.

[SC] R. G. Stanton and D. D. Cowan, Note on a 'square' functional equation, SIAM Review, 12 (1970), 277-279.

[T] A. Tucker, Applied Combinatorics, Wiley, New York, 1980.

Received July 19, 1985. The second author was supported by a grant from the Deutsche Forschungsgemeinschaft. The fifth author was supported by the National Science Foundation, by an Alfred P. Sloan Fellowship, and by an Arnold O. Beckman Fellowship at the UIUC Center for Advanced Study.

Hungarian ACADEMy of Sciences

BUDAPEST, HUNGARY

UNIVERSITY OF ILLINOIS

URBANA, IL 61801

AT \& T Bell LABORATORIES

MURRAY HiLl, NJ 07974

AND

UNIVERSITY OF ILLINOIS

URBANA, IL 61801 



\title{
PACIFIC JOURNAL OF MATHEMATICS \\ EDITORS
}

\section{S. VARADARAJAN}

(Managing Editor)

University of California

Los Angeles, CA 90024

HERBERT CLEMENS

University of Utah

Salt Lake City, UT 84112

R. FINN

Stanford University

Stanford, CA 94305
HERMANN FLASCHKA

University of Arizona

Tucson, AZ 85721

RAMESH A. GANGOLLI

University of Washington Seattle, WA 98195

VAughan F. R. Jones

University of California

Berkeley, CA 94720

ROBION KIRBY

University of California

Berkeley, CA 94720
C. C. MOORE

University of California

Berkeley, CA 94720

H. SAMELSON

Stanford University

Stanford, CA 94305

HAROLD STARK

University of California, San Diego

La Jolla, CA 92093

\section{ASSOCIATE EDITORS}

\author{
R. ARENS \\ E. F. BECKENBACH \\ B. H. NEUMANN \\ F. WOLF \\ K. YoshIDA \\ (1906-1982)

\section{SUPPORTING INS'TITUTIONS} \\ UNIVERSITY OF ARIZONA \\ UNIVERSITY OF BRITISH COLUMBIA \\ CALIFORNIA INSTITUTE OF TECHNOLOGY \\ UNIVERSITY OF CALIFORNIA \\ MONTANA STATE UNIVERSITY \\ UNIVERSITY OF NEVADA, RENO \\ NEW MEXICO STATE UNIVERSITY \\ OREGON STATE UNIVERSITY \\ UNIVERSITY OF OREGON \\ UNIVERSITY OF SOUTHERN CALIFORNIA \\ STANFORD UNIVERSITY \\ UNIVERSITY OF HAWAII \\ UNIVERSITY OF TOKYO \\ UNIVERSITY OF UTAH \\ WASHINGTON STATE UNIVERSITY \\ UNIVERSITY OF WASHINGTON
}

The Supporting Institutions listed above contribute to the cost of publication of this Journal, but they are not owners or publishers and have no responsibility for its content or policies.

Mathematical papers intended for publication in the Pacific Journal of Mathematics should be in typed form or offset-reproduced (not dittoed), double spaced with large margins. Please do not use built up fractions in the text of the manuscript. However, you may use them in the displayed equations. Underline Greek letters in red, German in green, and script in blue. The first paragraph must be capable of being used separately as a synopsis of the entire paper. In particular it should contain no bibliographic references. Please propose a heading for the odd numbered pages of less than 35 characters. Manuscripts, in triplicate, may be sent to any one of the editors. Please classify according to the scheme of Math. Reviews, Index to Vol. 39. Supply name and address of author to whom proofs should be sent. All other communications should be addressed to the managing editor, or Elaine Barth, University of California, Los Angeles, California 90024.

There are page-charges associated with articles appearing in the Pacific Journal of Mathematics. These charges are expected to be paid by the author's University, Government Agency or Company. If the author or authors do not have access to such Institutional support these charges are waived. Single authors will receive 50 free reprints; joint authors will receive a total of 100 free reprints. Additional copies may be obtained at cost in multiples of 50 .

The Pacific Journal of Mathematics is issued monthly as of January 1966. Regular subscription rate: $\$ 190.00$ a year (5 Vols., 10 issues). Special rate: $\$ 95.00$ a year to individual members of supporting institutions.

Subscriptions, orders for numbers issued in the last three calendar years, and changes of address should be sent to Pacific Journal of Mathematics, P.O. Box 969, Carmel Valley, CA 93924, U.S.A. Old back numbers obtainable from Kraus Periodicals Co., Route 100, Millwood, NY 10546.

The Pacific Journal of Mathematics at P.O. Box 969, Carmel Valley, CA 93924 (ISSN 0030-8730) publishes 5 volumes per year. Application to mail at Second-class postage rates is pending at Carmel Valley, California, and additional mailing offices. Postmaster: send address changes to Pacific Journal of Mathematics, P.O. Box 969, Carmel Valley, CA 93924.

PUBLISHED BY PACIFIC JOURNAL OF MATHEMATICS, A NON-PROFIT CORPORATION Copyright (c) 1987 by Pacific Journal of Mathematics 


\section{Pacific Journal of Mathematics}

\section{Vol. 126, No. 2 December, 1987}

Charles James Keith Batty, Derivations on the line and flows along orbits

Paul Erdốs, Adolf J. Hildebrand, Andrew Odlyzko, Paul Pudaite and

Bruce Reznick, The asymptotic behavior of a family of sequences . . . 227

Gregory James Galloway, A note on the fundamental group of a compact

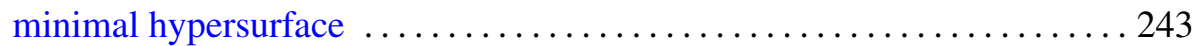

Nigel Higson, A characterization of $K K$-theory $\ldots \ldots \ldots \ldots \ldots \ldots \ldots \ldots 253$

Anthony To-Ming Lau and Wataru Takahashi, Weak convergence and nonlinear ergodic theorems for reversible semigroups of nonexpansive mappings

Pere Menal and Juame Moncasi, Lifting units in self-injective rings and an

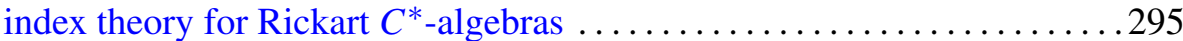

Yoshimi Saito, Schrödinger operators with a nonspherical radiation condition

Larry Smith, Realizing certain polynomial algebras as cohomology rings of

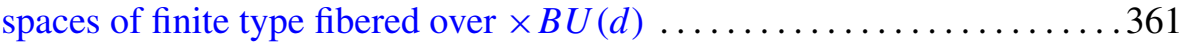

Carl E. Swenson and Calvin T. Long, Necessary and sufficient conditions for simple $A$-bases . . . . . . . . . . . . . . . . . . . . . . . . . . . . . . . 379

Kenneth Giovanni Valente, The $p$-primes of a commutative ring 385 